\title{
AADHAR based Electronic Voting Machine using Arduino
}

\author{
R. Murali Prasad, PhD \\ Professor \\ Dept. of ECE \\ Vardhaman College of Engg., \\ Hyderabad,
}

\author{
Polaiah Bojja, PhD \\ Professor \\ Dept. of ECE \\ K L University, Guntur
}

\author{
Madhu Nakirekanti \\ Assistant Professor \\ Dept. of ECE \\ Vardhaman College of Engg. \\ , Hyderabad
}

\begin{abstract}
This paper describes an online electoral system for Indian election is proposed for the first time. The voting system is managed in a easier way as all the users should login by Aadhar card number and password and click on his/her favorable candidates to cast the vote. This features a larger security in the sense that voter high security password is confirmed before the vote is accepted in the main database of ECI. The extra feature of the model is that the voter will ensure if his/her vote has gone to correct candidate/party. The votes are going to be done automatically, therefore saving an enormous time and facultative ECI to announce the result at intervals a very short period.
\end{abstract}

\section{Keywords}

Aadhar, Arduino Uno, Raspberry Pi, Finger Print, Voting System.

\section{INTRODUCTION}

This paper examines policy regarding the electronic approaches and developments towards electronic data storage and transmission. Finger print devices for voting machines and different existing identity documents are mentioned and enforced during this project.

The user should show his voter ID card whenever he goes to the booth to poll his vote. This is often a time consuming method because the person needs to check the voter ID card with the list he has, make sure it as an authorized card and then enable the person to poll his vote. Thus, to avoid this type of issues, designed a finger print based voting machine wherever the individuals no ought to carry his ID which contains his entire details. The person at the booth should show his Finger. This Finger print reader reads the details from the tag. This information is passed to the controlling unit for the verification. The controller reads DATA from the reader and compares this data with the already existing data. If the data matches with the already stored information, the person is allowed to poll his vote. If not, a message is displayed on LCD and therefore the person isn't allowed to poll his vote. The polling mechanism carries out manually using the switches. LCD is employed to display the related messages.

Voting is a method by which the electorates appoint their representatives. In current voting system the voter should show his voter ID card whenever an individual goes to the booth to poll one's vote. This process could be a time consuming method as the person needs to check the voter ID card with the list he has, confirm it as an authorized card and then enable the person to poll his vote. Thus, to avoid this type of problems, designed a finger print based mostly voting machine wherever the person no needs to carry his ID that contains his entire details.

The objective of voting is to permit voters to exercise their right to express their choices regarding specific issues, items of legislation, citizen initiatives, constitutional amendments, recalls and/or to decide on their government and political representatives. Technology is being employed additional and more as a tool to help voters to cast their votes. To permit the exercise of this right, the majority voting systems around the world include the following steps: citizen identification and authentication, voting and recording of votes cast, vote counting, publication of election results.

Voter identification is needed during two phases of the electoral process: first for voter registration so as to determine the right to vote and subsequently, at voting time, to allow a citizen to exercise their right to vote by verifying if the person satisfies all the necessities required to vote (authentication).

Security could be a heart of e-voting method. So the requirement of designing a secure e-voting system is very vital. Usually, mechanisms that ensure the security and privacy of an election are often time consuming, expensive for election administrators, and inconvenient for voters. There are completely different levels of e-voting security. So serious measures should be taken to keep it out of public domain. Also, security should be applied to hide votes from publicity. There's no measurement for acceptable security level, as a result of the extent depends on kind of the information. An appropriate security level is always a compromise between usability and strength of security method.

The secured e-voting process can be done by linking the voting machines with the Aadhar, an Indian citizen identification data base with a unique identification number for every citizen. The Aadhar based EVM can result in secured e-voting process. As a result of no two or more voter's data can match as this system uses biometrics.

Biometrics is the science and technology of measuring and analyzing biological data. In information technology, biometrics refers to technologies that measure and analyze human body characteristics, such as DNA, fingerprints, eye retinas and irises, voice patterns, facial patterns and hand measurements, for authentication purposes. During this paper used thumb impression for the purpose of voter identification or authentication. As the thumb impression of each individual is exclusive, it helps in maximising the accuracy.

Aadhar database is created containing the thumb impressions of all the voters in the constituency. Illegal votes and repetition of votes is checked for in this system. Hence if this system is utilized the elections would be truthful and free from rigging. 


\section{LITERATURE SURVEY}

\subsection{Issues of Existing Voting System}

Electronic Voting Machines ("EVM"), Idea mooted by the Chief Election Commissioner in 1977. The EVMs were devised and designed by Election Commission of India in collaboration with Bharat Electronics Limited (BEL), Bangalore and Electronics Corporation of India Limited (ECIL), Hyderabad. The EVMs are now manufactured by the above two undertakings. An EVM consists of two units,

\section{i) Control Unit \\ ii) Balloting Unit}

The two units are joined by a five-meter cable. The Control Unit is with the Presiding Officer or a Polling Officer and the Balloting Unit is placed inside the voting compartment.

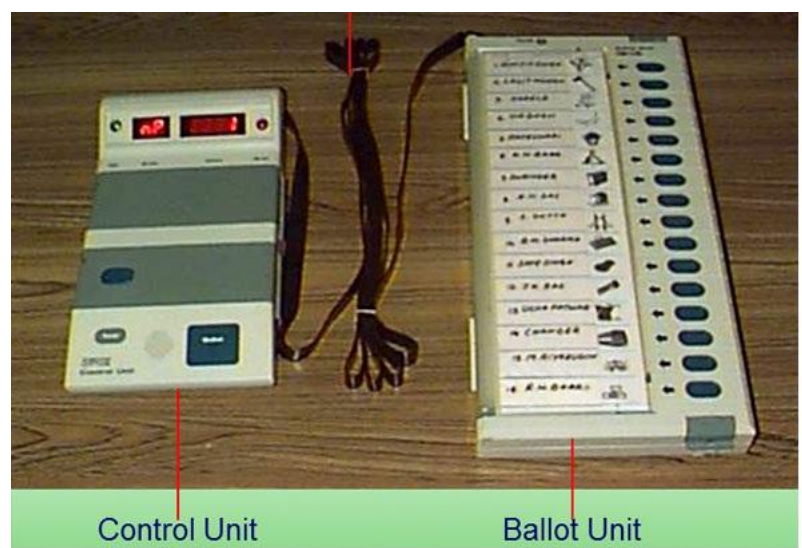

Fig 2.1 Sub-units of EVM

There are many types of problems with EVM which is currently in use they are:

1. Accuracy: It is not possible for a vote to be altered e laminated the invalid vote cannot be counted from the finally tally.

2. Democracy: It permits only eligible voters to vote and, it ensures that eligible voters vote only once.

3. Security Problems - One can change the program installed in the EVM and tamper the results after the polling. By replacing a small part of the machine with a look-alike component that can be silently instructed to steal a percentage of the votes in favor of a chosen candidate. These instructions can be sent wirelessly from a mobile phone.

4. Illegal Voting (Rigging) - The very commonly known problem Rigging which is faced in every electoral procedure. One candidate casts the votes of all the members or few amounts of members in the electoral list illegally. This results in the loss of votes for the other candidates participating and also increases the number votes to the candidate who performs this action. This can be done externally at the time of voting.
5. Privacy: Neither authority nor anyone else can link any ballot to the voter

6. Verifiability: Independently verification of that all votes have been counted correctly.

7. Resistance: No electoral entity (any server participating in the e lection) or group of entities, running the election can work in a conspiracy to introduce votes or to prevent voters from voting.

8. Availability: The system works properly as long as the poll stands and any voter can have access to it from the beginning to the end of the poll.

9. Resume Ability: The system allows any voter to interrupt the voting process to resume it or restart it while the poll stands. The existing elections were done in traditional way, using ballot, ink and tallying the votes later. But the proposed system prevents the election from being accurate.

\subsection{Securities of the Aadhar Based E- Voting system}

The main goal of a secure e-voting is to ensure the privacy of the voters and of the votes. A secure e-voting system are satisfies the following requirements,

1. Eligibility: only votes of legitimate voters shall be taken into account.

2. Anonymity: votes are set secret

3. Accuracy: cast ballot cannot be altered. Therefore, it must not be possible to delete ballots nor to add ballots, once the election has been closed.

4. Fairness: partial tabulation is impossible.

5. Vote and go: once a voter has casted their vote, no further action prior to the end of the election.

6. Public verifiability: anyone should be able to readily check the validity of the whole voting process.

\section{DESIGN AND IMPLEMENTATION}

In this technique, the details of the voter can get from the AADHAR card database. It was a newly developed information which has all the information concerning the people. By using this database took the voter's information will be stored within the personal computer. At the time of elections, for finger print accessing used finger sensing module.

Fingerprint verification could also be an honest choice for in e-voting systems, where you can provide users adequate explanation and training, and where the system operates in a controlled environment. it is not stunning that the workstation access application area looks to be based almost exclusively on finger prints, as a result of the relatively low price, small size, and easy integration of fingerprint authentication devices Capture the finger vein image and compare or match to database, capture finger vein and database finger vein matched suggests that this person will be valid for polling section and if condition is satisfied automatically. 


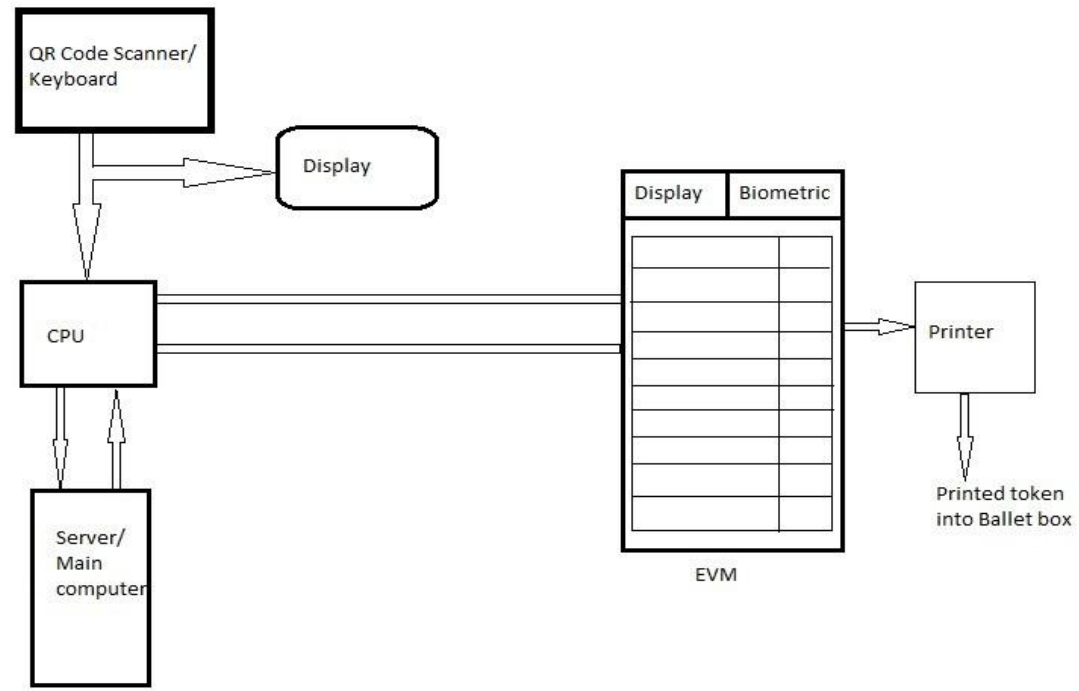

Fig 3.1 Block Diagram of Aadhar based EVM

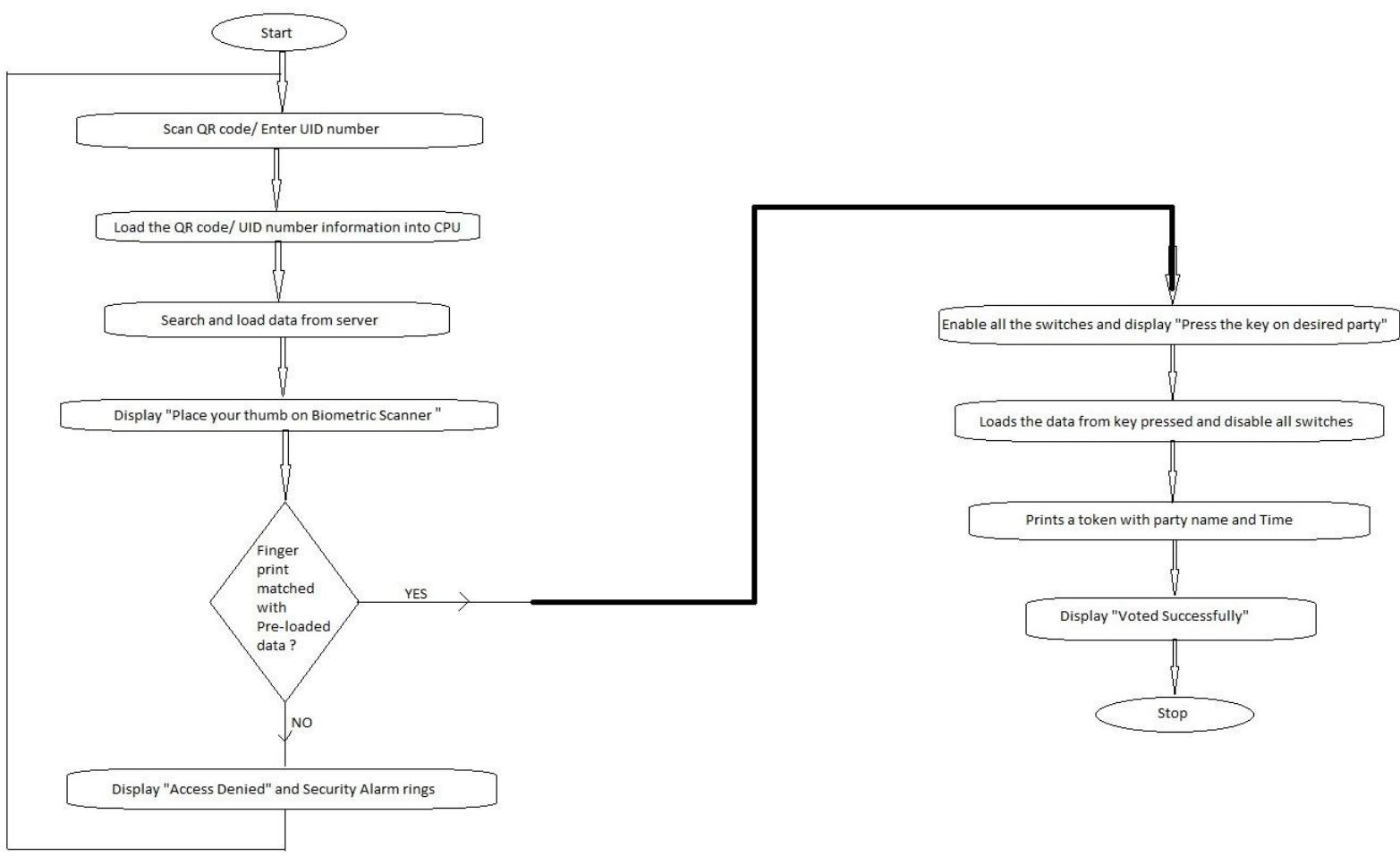

Fig 3.2 Flowchart

E-voting machine buttons will be activate otherwise deactivate buttons when the E-voting machine buttons square measure activated, the elector forged his/her vote. When completion of his/her ballot method, a "voting method completed" message are displayed on the screen. The amount of votes is counted by the E-Voting machine and therefore the data are sent to the Server through the online technology.

In earlier days the election process is in such a way that there will a box and a paper with all the political parties list. Whereas voting the voter has to put a stamp over the party symbol of his/her desired candidate in a specific consistency. This is an extended time consuming method and extremely a lot of prone to errors. Additionally the probabilities for rigging were a lot of during this traditional methodology. To beat of these ballot papers, stamps, boxes etc., going for Aadhar based EVM. So that, to beat time consumption, Rigging, insecurities etc.,Here in Aadhar primarily based EVM, using the information primarily based server for Aadhar details, Raspberry-pi for the online technology and arduino is employed for interfacing Raspberry-pi. 
The elector is allowed into election booth with Aadhar card ID. Here the voter first gives his Aadhar card for QR reading/Keypad operator. The elector is allowed into the ballot box room when the QR reading/UID authentication is finished with success by the operator. When Authentication the digital display in EVM displays as "welcome voter". The elector has to scan his thumb mistreatment the biometric and providing the thumb data that's scanned is matched with the pre-loaded server information the elector can permit to forge the vote.

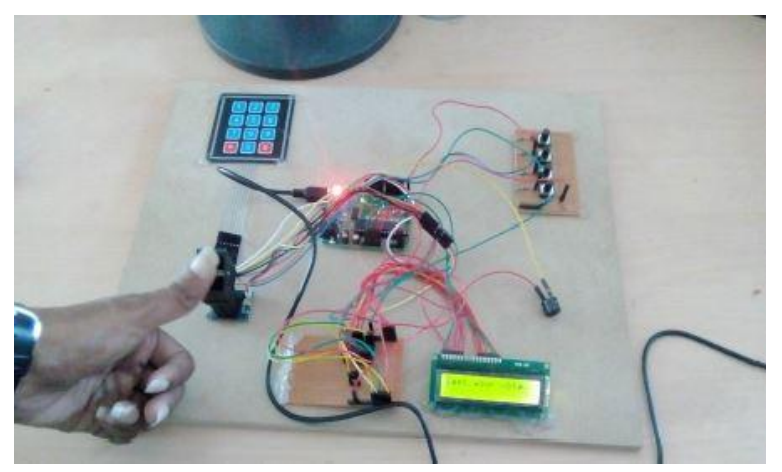

Fig 3.3 Voter Authentication

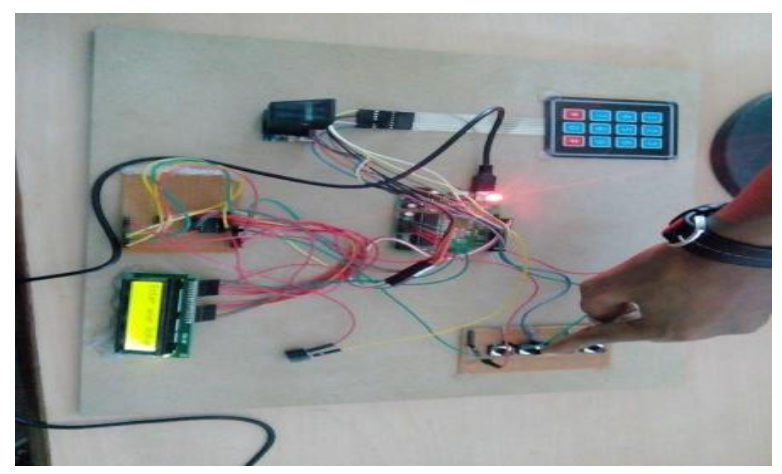

Fig 3.4Casting Vote

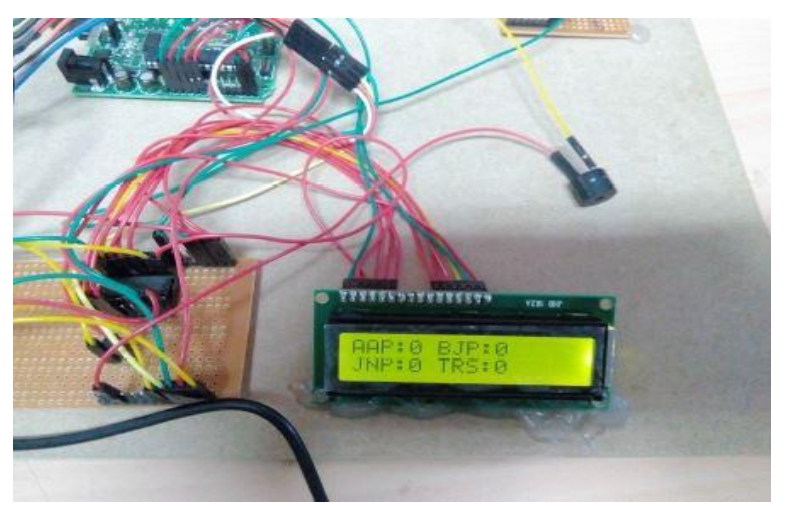

Fig 3.5 Final Results On LCD

Otherwise the Authentication will fail and voter will not be able to cast the vote. Once the voter is authenticated the switches of parties will enable and conjointly the data of elector are shown on show of EVM. When casting the vote the switches are disabled till next voter is authenticated and EVM shows a message as "thank you for voting". At identical time the printer connected to EVM can print the casted vote information at the side of a message "Please drop the token into ballot box". The token is additionally useful to verify the vote casted by the voter. And at last the voter will check the token and drop it into the box. The overall data of casted votes is distributed to the server mistreatment net technology in order that the results are often declared among all the consistencies.

\section{CONCLUSION}

This review discussed introduction about EVM and its variation, Issues of EVM, Taxonomy, and Biometric based EVM. Our efforts to understand electronic voting systems leave us optimistic, but concerned. This paper suggest that the EVM system has to be further studied and innovated to reach all level of community, so that the voter confidence will increase and election officials will make more involvement in purchasing the innovated EVM's for conduct smooth, secure, tamper- resistant Elections. This concludes that the Aadhar based EVM will useful

- To avoid Rigging

- To avoid time consumption

- To keep the voter's information more secured.

\section{REFERENCES}

[1] Ashok Kumar D., Ummal Sariba Begum T., "A Novel design of Electronic Voting System Using Fingerprint", International Journal of Innovative Technology \& Creative Engineering (ISSN:2045-8711),Vol.1,No.1. pp: 12 19, January 2011.

[2] Benjamin B., Bederson, Bongshin Lee., Robert M. Sherman., Paul S., Herrnson, Richard G. Niemi., "Electronic Voting System Usability Issues", In Proceedings of the SIGCHI conference on Human factors in computing systems, 2003.

[3] California Internet Voting Task Force. "A Report on the Feasibility of Internet Voting”, Jan.2000.

[4] Chaum D., "Secret-ballot receipts: True voter-verifiable elections", IEEE Security and Privacy38-47, 2004.

[5] Darcy, R., \& McAllister, I., "Ballot Position Effects", Electoral Studies, 9(1), pp.5-17, 1990.

[6] Dill D.L., Mercuri R., Neumann P.G., and Wallach D.S., "Frequently Asked Questions about DRE Voting Systems", Feb.2003.

[7] Gritzalis D., [Editor]., "Secure Electronic Voting", Springer-Verlag, Berlin Germany, 2003. 